The Astrophysical Journal, 608:1028-1038, 2004 June 20

(C) 2004. The American Astronomical Society. All rights reserved. Printed in U.S.A.

\title{
A CNO DICHOTOMY AMONG O2 GIANT SPECTRA IN THE MAGELLANIC CLOUDS
}

\author{
Nolan R. WaLbORN \\ Space Telescope Science Institute, ${ }^{1} 3700$ San Martin Drive, Baltimore, MD 21218; walborn@stsci.edu \\ Nidia I. MorrelL ${ }^{2,3}$ \\ Las Campanas Observatory, Carnegie Observatories, Casilla 601, La Serena, Chile; nmorrell@oveja.lco.cl \\ IAN D. HOWARTH \\ Department of Physics and Astronomy, University College London, Gower Street, London WC1E 6BT, UK; idh@star.ucl.ac.uk \\ Paul A. Crowther ${ }^{4}$ \\ Department of Physics and Astronomy, University of Sheffield, Hounsfield Road, Sheffield S3 7RH, UK; paul.crowther@sheffield.ac.uk \\ DANIEL J. LENNON \\ Isaac Newton Group of Telescopes, Apartado de Correos 321, 38700 Santa Cruz de La Palma, Canary Islands, Spain; dj1@ing.iac.es \\ Philip Massey ${ }^{2}$ \\ Lowell Observatory, 1400 West Mars Hill Road, Flagstaff, AZ 86001; massey@lowell.edu \\ AND \\ Julia I. ARIAs ${ }^{2,5}$ \\ Facultad de Ciencias Astronómicas y Geofísicas, Universidad Nacional de La Plata, Paseo del Bosque s/n, \\ 1900 La Plata, Argentina; julia@lahuan.fcaglp.unlp.edu.ar \\ Received 2003 October 13; accepted 2004 March 1
}

\begin{abstract}
From a survey of the $3400 \AA$ region in the earliest O-type spectra, we have found that two of the four O2 giants observed in the Large Magellanic Cloud have O Iv lines there that are stronger than the $\mathrm{N}$ IV lines, while the other two have the opposite. A Small Magellanic Cloud counterpart also has N IV stronger than O IV. Inspection of the blue spectra of these stars shows that the former pair have weaker $\mathrm{N}$ lines in all ionization states (III, IV, and v) present as well as lines of C IV $\lambda 4658$, while the latter three have stronger $\mathrm{N}$ lines and greater $\mathrm{He} / \mathrm{H}$. Space ultraviolet observations of two of the N-strong stars show $\mathrm{N} v$ wind profiles substantially stronger than those of $\mathrm{C}$ IV, while in the $\mathrm{N}$-weak stars the $\mathrm{C}$ IV features are equal to or stronger than the $\mathrm{N} v$. The $\mathrm{N}$-strong stars are now reclassified as ON2 III( $\left.\mathrm{f}^{*}\right)$, newly defining that category. These characteristics strongly suggest a larger fraction of processed material in the atmospheres of the ON2 stars, which we confirm by modeling the optical spectra. In the context of current models, it is in turn implied that the ON2 stars are in a more advanced evolutionary state than the others, and/or that they had higher initial rotational velocities. The recent formulation of the effects of rotation on massive stellar evolution introduces an additional fundamental parameter, which the CNO abundances are in principle able to constrain. We present some illustrative comparisons with current Geneva evolutionary models for rotating massive stars. It is possible that these very hot, nitrogen-rich objects are products of homogeneous evolution. Our results will provide motivation for further physical modeling of the atmospheres and evolutionary histories of the most massive hot stars.
\end{abstract}

Subject headings: Magellanic Clouds — stars: abundances — stars: early-type — stars: evolution — stars: fundamental parameters

\section{INTRODUCTION}

Anomalies in the relative intensities of $\mathrm{CNO}$ lines in $\mathrm{OB}$ spectra have been described for some time (Walborn 1976, 1988, 2003; and references therein). Anticorrelations of $\mathrm{N}$ versus $\mathrm{C}$ and $\mathrm{O}$ and correlations with $\mathrm{He} / \mathrm{H}$ in the expected

\footnotetext{
${ }^{1}$ Operated by the Association of Universities for Research in Astronomy, Inc., under NASA contract NAS5-26555.

2 Visiting Astronomer, Cerro Tololo Inter-American Observatory, National Optical Astronomy Observatory, operated by the Association of Universities for Research in Astronomy, Inc., under a cooperative agreement with the NSF.

${ }^{3}$ Member of the Carrera del Investigador Científico, CONICET, Argentina; on leave from Facultad de Ciencias Astronómicas y Geofísicas, Universidad Nacional de La Plata.

${ }^{4}$ Visiting Astronomer, European Southern Observatory Very Large Telescope; program 70.D-0164.

5 Fellow of CONICET, Argentina.
}

sense have encouraged interpretations in terms of mixing of $\mathrm{CNO}$-cycled material into the atmospheres and winds of massive stars. An important point has been the recognition that the morphologically normal majority of OB supergiants actually have some degree of $\mathrm{N}$ and He enhancements already, while the OBC minority have normal (i.e., main-sequence) abundances, and the OBN group have more extreme mixing (Maeder \& Conti 1994; Smith \& Howarth 1994). The recent development of models for massive stellar evolution with rotation (Maeder \& Meynet 2000) is an advance toward realism, since massive stars often rotate rapidly, but another dimension is added to the phase space that must be explored in order to compare observations with theory. Fortunately, a new observational diagnostic is added concurrently, namely, the CNO abundances, since the degree of mixing also depends on the initial rotation. Indeed, progress has already been 
made toward understanding apparent discrepancies among the Hertzsprung-Russell diagram (HRD) locations of some mid-O giants and supergiants in the Small Magellanic Cloud (SMC; Walborn et al. 2000) in terms of their CNO abundances and inferred initial rotations (Crowther et al. 2002; Hillier et al. 2003). Related results for main-sequence stars in the SMC giant $\mathrm{H}$ II region NGC 346 are reported by Bouret et al. (2003).

Spectral classification of the hottest massive stars is relatively difficult because of their scarcity and the paucity of reliable features in their optical spectra. Recently, an increased sample of high-quality data has supported classification developments for them, including introduction of the new types $\mathrm{O} 2$ and $\mathrm{O} 3.5$ (Walborn et al. 2002). In this system, reliance is placed on ratios of the selective emission lines (Walborn 2001) of $\mathrm{N}$ IV and $\mathrm{N}$ III, primarily on an empirical basis. These new spectral types have yet to be modeled systematically and calibrated in terms of physical parameters, including confirmation that the $\mathrm{O} 2$ extension corresponds to higher effective temperatures as hypothesized. The analysis of these spectra is also difficult because of uncertainties in the parameters and the atmospheric physics. For example, the special behavior of the $\mathrm{N}$ IV and $\mathrm{N}$ III emission lines has to be reproduced and interpreted. Currently, modeling and calibration developments are under way to address these issues; we present some relevant results here.

Certainly additional spectral features to constrain the classification and analysis of the hottest spectra would be welcome. Drissen et al. (1995) pointed out certain little-observed $\mathrm{N}$ IV and $\mathrm{O}$ IV lines in the $3400 \AA$ region of early O spectra that warranted further investigation in a larger sample including longer wavelength classification standards. We have now accomplished such a survey, which will be fully reported by N. Morrell et al. (2004, in preparation). Here we describe our unanticipated discovery of the first $\mathrm{CNO}$ anomalies in optical O2 spectra, which are relevant to both the classification and modeling of these extreme objects.

\section{OBSERVATIONS}

The far-violet observations of the Large Magellanic Cloud (LMC) stars were carried out with the Cassegrain spectrograph at the Cerro Tololo Inter-American Observatory (CTIO) $1.5 \mathrm{~m}$ telescope during 2002 February 22-27. A 600 lines $\mathrm{mm}^{-1}$ grating blazed at $3375 \AA$ in second order combined with a Loral $1 \mathrm{k}$ CCD provided a 2 pixel resolution of $1.5 \AA$ and wavelength coverage from 3270 to $4180 \AA$. The LMC stars discussed here have $(V, B-V)$ ranging from (12.28, $-0.23)$ for HDE 269810 to $(13.68,-0.01)$ for $\mathrm{LH} 10-3061$ (see Walborn et al. 2002 for detailed stellar parameters). Total exposure times ranging from 1 to $3 \mathrm{hr}$ yielded signal-to-noise values between 60 and 150 pixel $^{-1}$ at $3400 \AA$, reflecting the low (but relatively high at CTIO) atmospheric transmission at that wavelength. The data for all but HDE 269810 have been smoothed by 3 pixels for presentation here, and the radial velocities have been corrected to rest. The data reductions and analysis were performed with standard $\mathrm{IRAF}^{6}$ routines running under Linux at the La Plata Observatory.

NGC 346-3 $(13.50,-0.23)$ in the SMC was observed on the night of 2003 July 30-31 with the Boller \& Chivens spectrograph at the Las Campanas Observatory (LCO) Magellan I (Walter Baade) $6.5 \mathrm{~m}$ telescope. The detector was a

\footnotetext{
${ }^{6}$ IRAF is distributed by the National Optical Astronomy Observatory.
}

2048 pixel $\times 515$ pixel Marconi CCD (pixel size $13.5 \mu \mathrm{m}$ ). A 600 lines $\mathrm{mm}^{-1}$ grating blazed at $5000 \AA$ provided wavelength coverage from 3300 to $6400 \AA$ with a 2 pixel resolution of $3.1 \AA$. The exposure time was 10 minutes, producing a signal-to-noise ratio of about 50 pixel $^{-1}$ near both ends of the spectrogram but reaching 300 at $5000 \AA$. The data were reduced and analyzed with standard IRAF routines at LCO.

Details of the blue-violet observations shown here can be found in Walborn et al. (2002). The ultraviolet observations discussed correlatively are from the Hubble Space Telescope (HST) Faint Object Spectrograph (FOS) and Space Telescope Imaging Spectrograph (STIS); they are fully described by Walborn et al. (1995) and by Massey et al. (2004), respectively.

As a check on the quantitative analysis of these moderateresolution data, an available high-resolution observation of HDE 269810 was also analyzed. This observation was made at the European Southern Observatory (ESO) Very Large Telescope (VLT), with the UV-Visual Echelle Spectrograph (UVES) mounted at the UT2 $8 \mathrm{~m}$, on 2002 November 29 under program 70.D-0164. The resolving power of this system is 70,000, providing a resolution of $0.05 \AA$ at the O IV $\lambda 3400$ lines. Two setups with several channels containing EEV and $\mathrm{MIT} / \mathrm{LL} 2 \mathrm{k} \times 4 \mathrm{k}$ CCDs with $15 \mu \mathrm{m}$ pixels covered wavelengths from the far violet through $\mathrm{H} \alpha$. Exposure times of $1200 \mathrm{~s}$ yielded a signal-to-noise ratio per 2 pixel resolution element ranging from 80 at $3450 \AA$ to 150 at $4700 \AA$ and 120 at $6600 \AA$. The data were reduced by the UVES pipeline under the Munich Image Data Analysis System (MIDAS).

\section{RESULTS}

A sample of 27 Galactic and Magellanic Cloud early O stars has been observed in the $3400 \AA$ region; in most of them, the $\mathrm{N}$ IV and $\mathrm{O}$ Iv features have comparable intensities and display systematic trends with spectral type and luminosity class, which will be discussed by N. Morrell et al. (2004, in preparation). Surprisingly, however, the four LMC O2 III(f*) spectra in the sample separate into two groups, with $\mathrm{O}$ IV substantially stronger than $\mathrm{N}$ IV in one pair and the opposite in the other, while the SMC object is similar to the latter LMC pair, as shown in Figure 1. This unexpected result prompted us to re-examine in a new light the blue-violet spectra of the same five stars as shown by Walborn et al. (2002) and reproduced in Figure 2. It was immediately clear that the systematic differences between HDE 269810 and Sk $-68^{\circ} 137$ on the one hand and LH 64-16, LH 10-3061, and NGC 346-3 on the other can be described consistently in terms of different relative line strengths of $\mathrm{N}$ versus $\mathrm{C}$. In particular, the first pair have weaker $\mathrm{N}$ III and $\mathrm{N}$ IV emission lines as well as $\mathrm{N} v$ absorption lines, along with the presence of $\mathrm{C}$ IV $\lambda$ 24658, while all of the $\mathrm{N}$ lines are stronger in the latter group (except for the $\mathrm{N} v$ in $\mathrm{NGC} \mathrm{346-3).} \mathrm{Moreover,} \mathrm{the} \mathrm{He} / \mathrm{H}$ line ratios are seen to be larger in the latter group as well (although unclear in the SMC object because of nebular hydrogen emission and the lower resolution in the far violet). Presumably the $\mathrm{O}$ IV $\lambda 4632$, which is also prominent in the first pair, is blended with $\mathrm{N}$ III $\lambda 4634$ in the other stars. The morphology strongly excludes ionization effects as an explanation and equally strongly suggests $\mathrm{N} /(\mathrm{C}, \mathrm{O})$ and $\mathrm{He} / \mathrm{H}$ abundance differences as the cause.

Striking support for this conjecture is provided by the ultraviolet stellar-wind spectra of these stars. Those of HDE 269810, Sk $-68^{\circ} 137$, and NGC 346-3 were discussed by Walborn et al. (1995) and are reproduced in Figure 3, along 


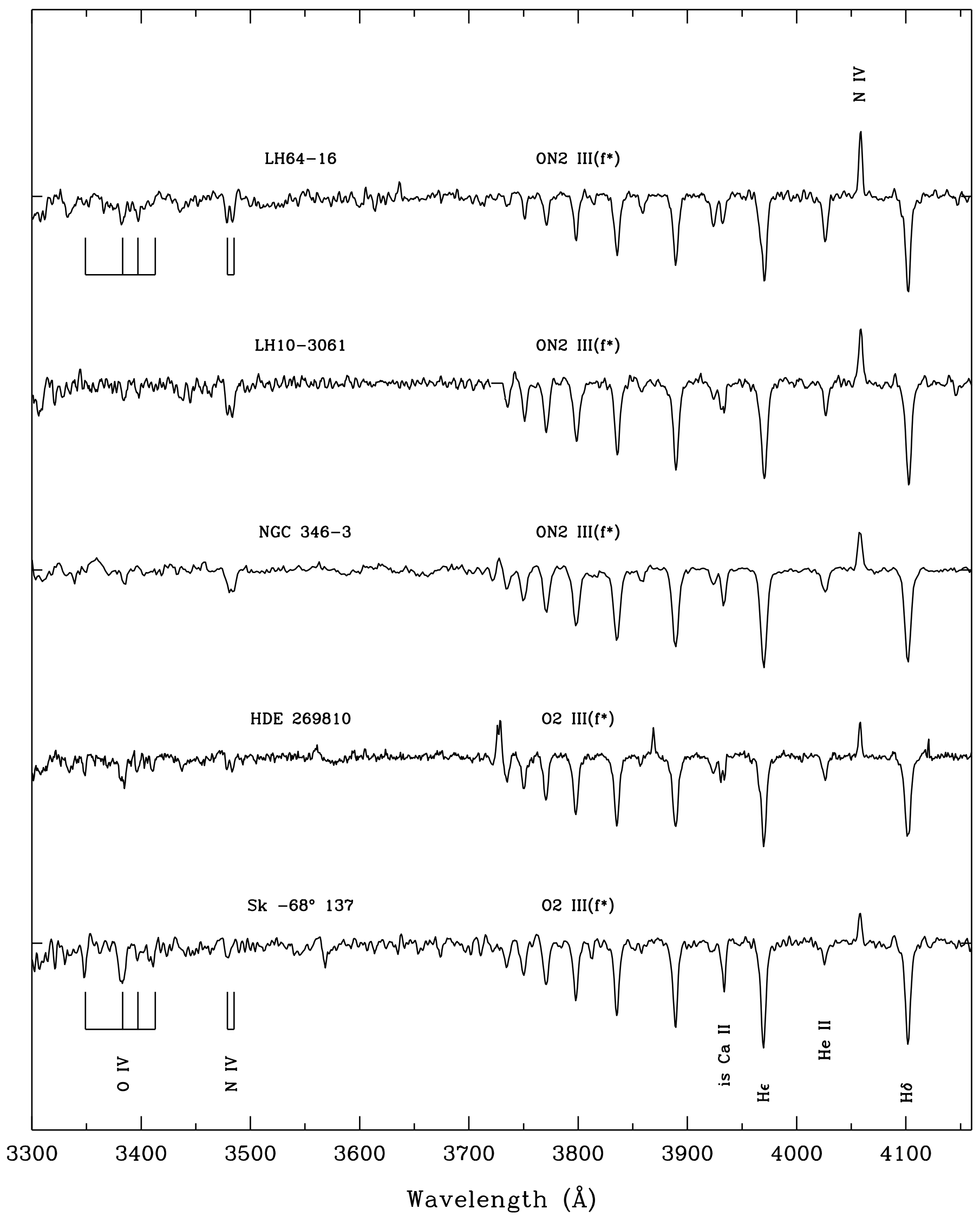

FIG. 1.-Rectified linear-intensity, far-violet spectrograms of five $\mathrm{O} 2$ giants in the Magellanic Clouds, separated by 0.5 continuum units. The absorption lines

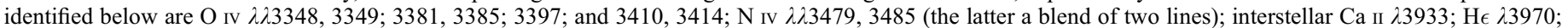

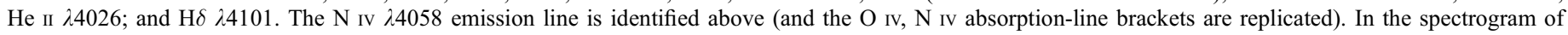

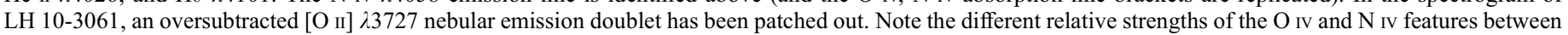
the $\mathrm{ON} 2$ and $\mathrm{O} 2$ classes. 


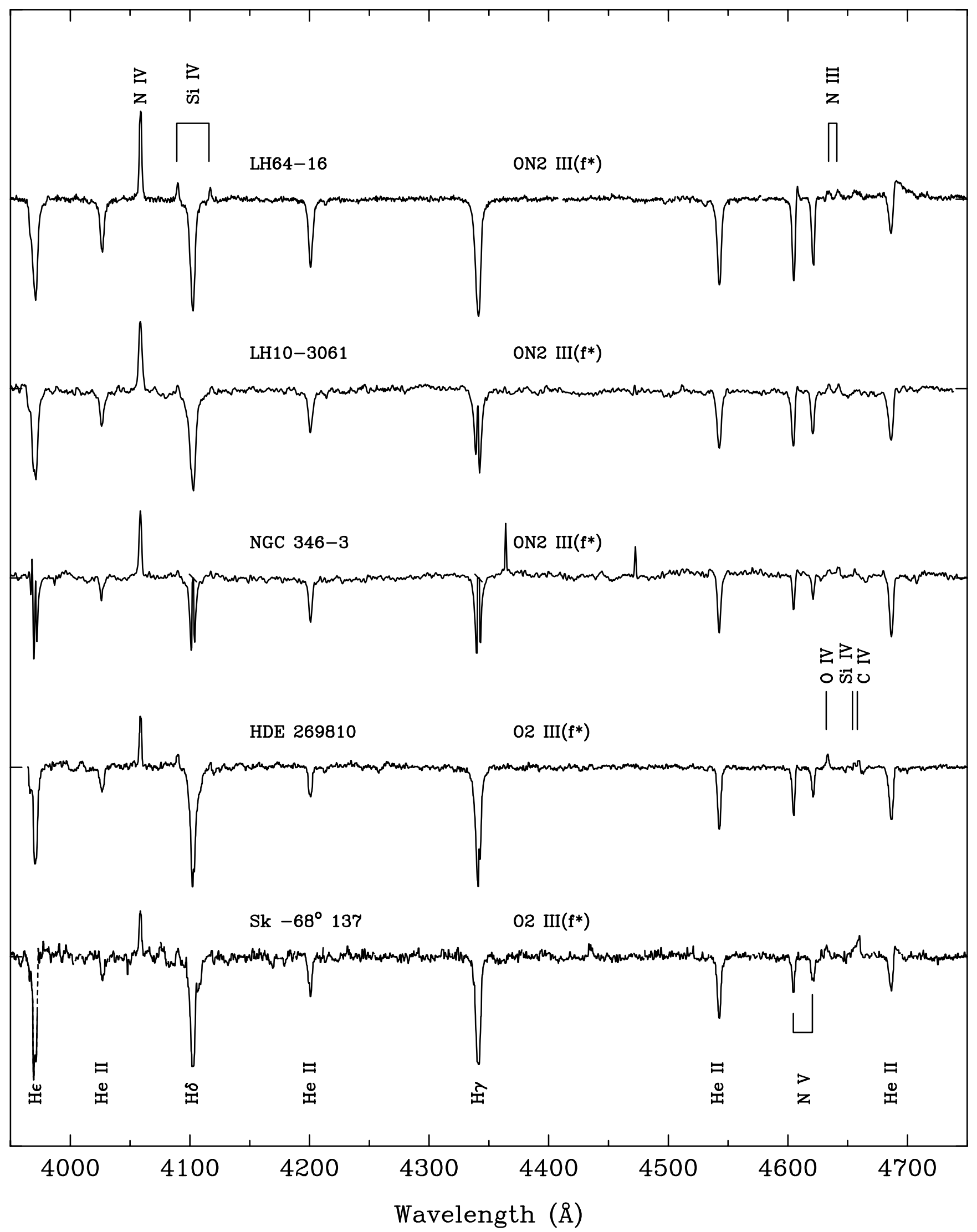

FIG. 2.-Blue-violet spectrograms of the same stars and with the same format as in Fig. 1. The absorption lines identified below are H $\epsilon$ $\lambda 3970, \mathrm{H} \delta \lambda 4101$, and $\mathrm{H} \gamma$ $\lambda 4340 ;$ He II $\lambda \lambda 4026,4200,4541,4686$; and $\mathrm{N} v \lambda \lambda 4604,4620$. At the top, N IV $\lambda 4058$; Si IV $\lambda \lambda 4089,4116$; and N III $\lambda \lambda 4634,4640,4642$ emission lines are identified. Above HDE 269810 , O Iv $\lambda 4632$, Si Iv $\lambda 4654$, and C IV $\lambda 4658$ emission lines are identified; this $\mathrm{O}$ Iv line had been identified with Si IV $\lambda 4631$ by Walborn et al. (2002), but we have now definitively established from its wavelength and intensity in high-resolution data (Fig. 5) that the O IV contribution is dominant. Hydrogen nebular emission lines have been truncated in NGC 346-3. Note the different N/C and He/H line ratios between the ON2 and O2 classes (the latter as permitted by nebular $\mathrm{H}$ emission contamination). 


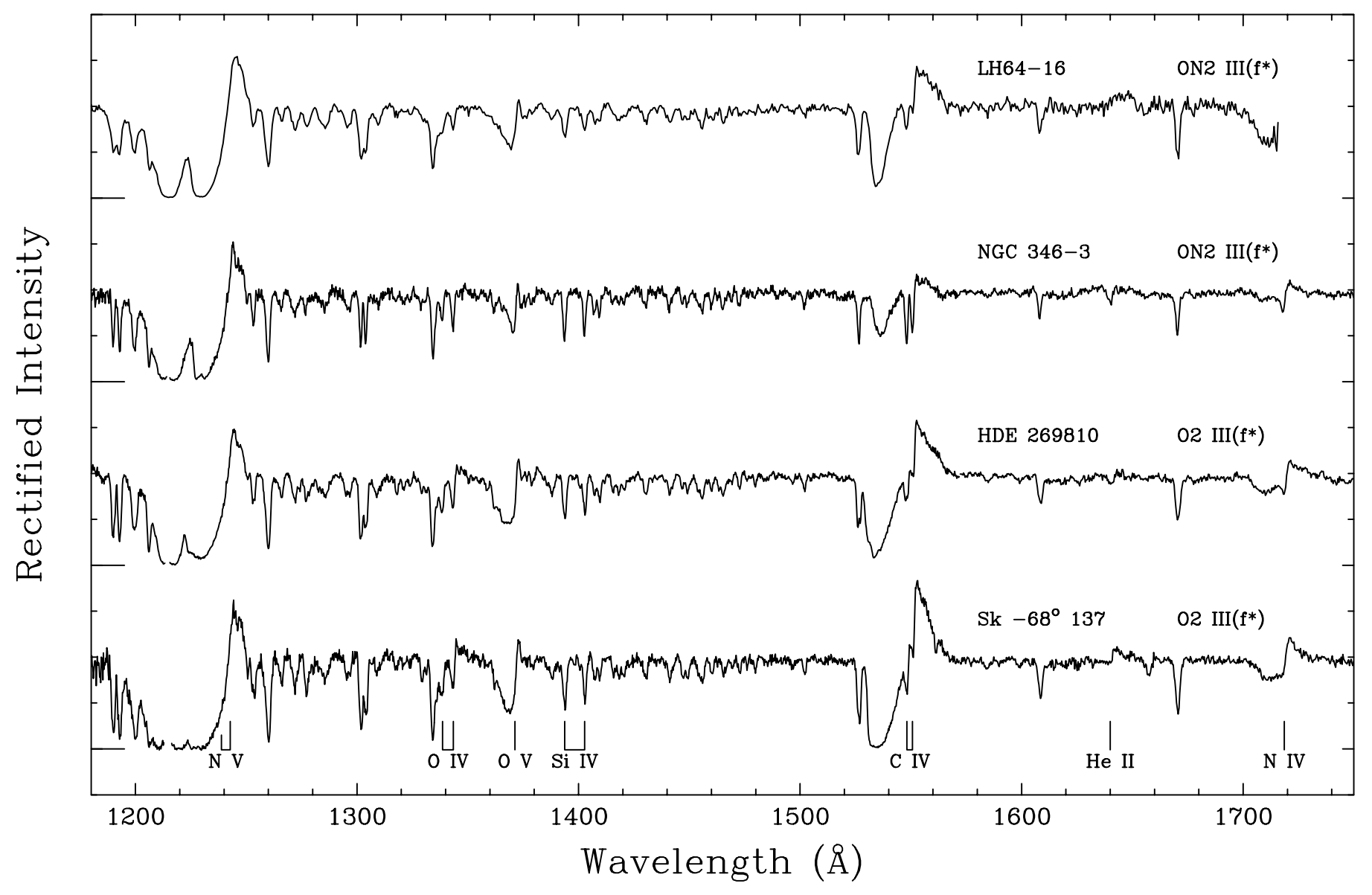

FIG. 3. - HST far-ultraviolet spectrograms of four stars shown in the previous figures. The longer ordinate tick marks give the zero levels, and the continua are normalized to 1.0. The spectral features identified below are $\mathrm{N} v \lambda \lambda 1239,1243$, O Iv $\lambda \lambda 1338,1343, \mathrm{O} \vee \lambda 1371$, Si rv $\lambda \lambda 1394,1403$ (interstellar), C iv $\lambda \lambda 1548,1551$, He II $\lambda 1640$, and $\mathrm{N}$ IV $\lambda 1718$. Note the difference between the relative strengths of the $\mathrm{N} v$ and $\mathrm{C}$ iv features between the upper and lower pairs of spectra; the $\mathrm{O}$ IV and $\mathrm{O} \vee$ features also appear weaker in the $\mathrm{ON} 2$ spectra.

with that of LH 64-16, which was obtained and analyzed by Massey et al. (2004). In HDE 269810, the C IV $\lambda 1550$ wind profile is comparable to that of $\mathrm{N} v \lambda 1240$, and in $\mathrm{Sk}-68^{\circ} 137$ the $\mathrm{C}$ IV is actually somewhat stronger than the $\mathrm{N} v$, while in LH $64-16 \mathrm{~N} v$ is stronger, and in NGC 346-3 much stronger, than C IV. The UV spectrum of LH 10-3061 has not yet been observed to our knowledge.

Both the optical and UV spectra of LH 64-16 and NGC 346-3, as well as LH 10-3061 in the optical, relative to HDE 269810 and $\mathrm{Sk}-68^{\circ} 137$, are consistent with the characteristics of the ON class (Walborn 1976, 1988, 2003). Hence, the first three spectra are hereby reclassified as ON2 III(f*). This class has been interpreted in terms of mixing of CNO-cycled material into the atmospheres and winds of the stars (e.g., Maeder \& Conti 1994). The presence of these effects at the earliest spectral types is a new development that may further challenge the models, however, as discussed below.

Walborn et al. (2000) had already classified NGC 346-3 as ON3 III(f*) purely on the basis of the very large $\mathrm{N}$ v/C IV wind-profile ratio in its UV spectrum. The $\mathrm{ON}$ designation was not retained when this star was moved into the $\mathrm{O} 2$ class by Walborn et al. (2002), partly because of concerns about a pure UV classification criterion and about the possibility that all such spectra might have similarly large N/C, obvious only in the SMC object because the lower systemic metallicity allowed the very sensitive $\mathrm{C}$ IV feature to desaturate. However, LH 64-16 demonstrates that the latter concern is not valid, since the $\mathrm{C}$ IV can be weaker than $\mathrm{N} v$ at LMC metallicity. Thus, the proper classification of NGC $346-3$ is also ON2 III(f*), as confirmed here by the $3400 \AA$ observations. Haser et al. (1998) derived a very large N/C abundance ratio in NGC $346-3$, but normal in Sk $-68^{\circ} 137$, while Puls et al. (1996) gave normal $\mathrm{He} / \mathrm{H}$ in both HDE 269810 and $\mathrm{Sk}-68^{\circ} 137$, consistent with the present classifications and interpretations. Bouret et al. (2003) have also derived an enhanced $\mathrm{N}$ abundance in NGC 346-3, as further discussed below.

\section{SPECTROSCOPIC MODELING}

In order to gain physical insight into the CNO line-strength differences outlined above, we have undertaken spectroscopic modeling of our blue and violet spectrograms. Example fits to the spectra of two stars are shown in Figure 4.

\subsection{Techniques}

Recent developments in model-atmosphere studies of hot stars routinely permit sophisticated metal-line blanketing in non-LTE to be considered under either plane-parallel geometry (e.g., TLUSTY; Lanz \& Hubeny 2003) or spherical geometry (e.g., CMFGEN; Hillier \& Miller 1998). Bouret et al. (2003) have demonstrated the excellent consistency in synthetic spectra achieved between TLUSTY and CMFGEN in a combined UV and optical study of NGC 346-3, ON2 III(f*). The former technique provides a more comprehensive treatment 

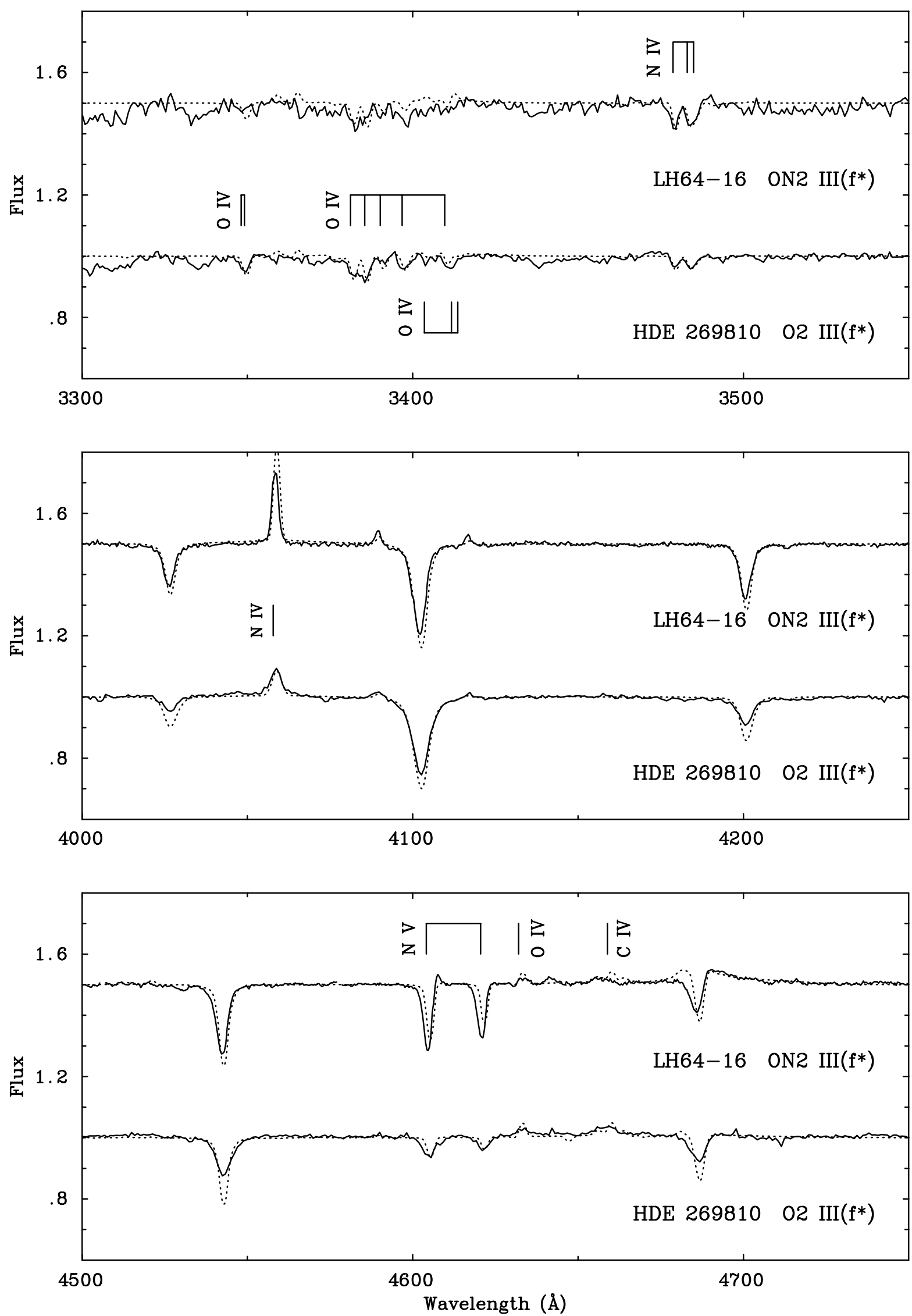

FIg. 4.-Atmospheric model fits (dotted lines) to key diagnostic features in two of the intermediate-resolution spectrograms (solid lines). The $56 \mathrm{kK}$ fit to LH 64 16 is shown (see text). The spectral lines identified in the top panel are $\mathrm{O}$ iv $\lambda \lambda 3348,3349 ; 3381,3385,3390,3397,3410 ; 3404,3412,3414$; and $\mathrm{N}$ iv $\lambda \lambda 3479,3483$, 3485. In the middle panel, $\mathrm{N}$ Iv $\lambda 4058$ is identified, and in the bottom panel, $\mathrm{N}$ v $\lambda \lambda 4604,4620, \mathrm{O}$ IV $\lambda 4632$, and $\mathrm{C}$ IV $\lambda 4658$. 
TABLE 1

Parameters of Analyzed Stars

\begin{tabular}{|c|c|c|c|c|c|}
\hline Parameter & HDE 269810 & Sk $-68^{\circ} 137$ & LH $64-16$ & LH 10-3061 & NGC $346-3$ \\
\hline Spectral Type .......................... & O2 III(f*) & O2 III(f*) & ON2 III(f*) & ON2 III(f*) & ON2 III(f*) \\
\hline$T_{\text {eff }}(\mathrm{kK})$ & 52.5 & 55 & 55 & 55 & 52.5 \\
\hline $\log L\left(L_{\odot}\right)$ & 6.34 & 6.19 & 5.90 & 6.08 & 6.07 \\
\hline $\log \dot{M}\left(M_{\odot} \mathrm{yr}^{-1}\right) \ldots \ldots \ldots \ldots \ldots$ & -5.6 & -5.7 & -5.8 & -6.0 & -5.8 \\
\hline$v_{\infty}\left(\mathrm{km} \mathrm{s}^{-1}\right)$ & $3750^{\mathrm{a}}$ & $3200^{\mathrm{b}}$ & $3250^{c}$ & $3200:^{b}$ & $2800^{\mathrm{d}}$ \\
\hline$M_{V}(\mathrm{mag})^{\mathrm{e}}$ & -6.6 & -6.1 & -5.4 & -5.8 & -5.9 \\
\hline$\xi\left(\mathrm{km} \mathrm{s}^{-1}\right)$ & 10 & 15 & 10 & 15 & 25 \\
\hline $\mathrm{He} / \mathrm{H}$ & $\leq 0.1$ & $0.1:$ & 0.25 & 0.1: & 0.1 \\
\hline $\mathrm{C} / \mathrm{C}_{\odot}(\mathrm{ISM})$ & $0.2(0.33)$ & $0.2(0.33)$ & $0.05(0.33)$ & $0.05(0.33)$ & $0.025(0.16)$ \\
\hline $\mathrm{N} / \mathrm{N}_{\odot}(\mathrm{ISM})$ & $0.4(0.17)$ & $0.3(0.17)$ & $1.2(0.17)$ & $1.2(0.17)$ & $0.4(0.05)$ \\
\hline $\mathrm{O} / \mathrm{O}_{\odot}(\mathrm{ISM})$ & $0.4(0.49)$ & $0.6(0.49)$ & $0.25(0.49)$ & $0.15(0.49)$ & $0.15(0.23)$ \\
\hline$(\mathrm{N} / \mathrm{C}) /(\mathrm{N} / \mathrm{C})_{\odot}(\mathrm{ISM}) \ldots \ldots \ldots . .$. & $2.0(0.52)$ & $1.5(0.52)$ & $24(0.52)$ & $24(0.52)$ & $16(0.31)$ \\
\hline$(\mathrm{N} / \mathrm{O}) /(\mathrm{N} / \mathrm{O})_{\odot}(\mathrm{ISM}) \ldots \ldots \ldots$ & $1.0(0.35)$ & $0.5(0.35)$ & $4.8(0.35)$ & $8.0(0.35)$ & $2.7(0.22)$ \\
\hline$(\mathrm{N} / \mathrm{C}) /(\mathrm{N} / \mathrm{C})_{\mathrm{ISM}} \ldots \ldots \ldots \ldots . .$. & 3.8 & 2.9 & 46 & 46 & 52 \\
\hline$(\mathrm{N} / \mathrm{O}) /(\mathrm{N} / \mathrm{O})_{\mathrm{ISM}} \ldots \ldots \ldots \ldots \ldots$ & 2.9 & 1.4 & 14 & 23 & 12 \\
\hline
\end{tabular}

${ }^{\mathrm{a}}$ From Walborn et al. (1995).

${ }^{\mathrm{b}}$ From Prinja \& Crowther (1998).

${ }^{\mathrm{c}}$ From Massey et al. (2004).

${ }^{d}$ From Bouret et al. (2003).

e From Walborn et al. (2002).

of photospheric metal-line blanketing, while the latter offers insights into wind properties.

In the present application, we have utilized CMFGEN, the current version of which is discussed by Hillier et al. (2003). The code solves the radiative transfer equation in the comoving frame under the additional constraint of statistical equilibrium. The model used here is similar to that applied by Bouret et al. (2003), except that some important additional ions are considered. In total, 27 ions of $\mathrm{H}, \mathrm{He}, \mathrm{C}, \mathrm{N}, \mathrm{O}, \mathrm{Si}, \mathrm{P}$, $\mathrm{S}, \mathrm{Ar}, \mathrm{Fe}$, and $\mathrm{Ni}$ are included, comprising a total of 2000 individual levels (grouped into 800 superlevels), with a full array of 17,000 bound-bound transitions. The temperature structure is determined by radiative equilibrium. CMFGEN does not solve the momentum equation, so a density or velocity structure is required. For the supersonic part, the velocity is parameterized with a classical $\beta$-type law (with $\beta=1$ ), which is connected to a hydrostatic density structure at depth such that the velocity and velocity gradient match at the interface. The subsonic velocity structure is set by the corresponding fully line-blanketed TLUSTY (ver. 200) model with $\log g=4.0 \mathrm{cgs}$.

We have assumed a depth-independent Doppler profile for all lines when solving for the atmospheric structure in the comoving frame, but in the final calculation of the emergent spectrum in the observer's frame, we have adopted a radially dependent turbulence, which reflects the effect of shocks due to wind instabilities. The minimum turbulence, $\xi$, is set from the core strengths of $\mathrm{H} \gamma$ and $\mathrm{He}$ II $\lambda$ 4541. Incoherent electron scattering and Stark broadening for hydrogen and helium lines are adopted. We convolve our synthetic spectrum with a rotational broadening profile. Because of the intermediate dispersion of our main observations, instrumental effects prevent a reliable determination of $v \sin i$, so $100 \mathrm{~km} \mathrm{~s}^{-1}$ has been adopted in each case, in accord with previous results for HDE 269810, Sk $-68^{\circ} 137$, and NGC 346-3 (Puls et al. 1996).

Temperatures of $\mathrm{O}$ stars are generally derived from spectroscopic fits to lines of He I-II (e.g., Conti 1973; Crowther et al. 2002). Since He $\mathrm{I}$ is absent or extremely weak in $\mathrm{O} 2$ stars, we rely instead on the $\mathrm{N} \mathrm{IV-V}$ ionization balance, i.e., $\mathrm{N}$ IV $\lambda \lambda 3479-3485,4058$ and $\mathrm{N} v \lambda \lambda 4604,4620$. Of course, the nitrogen abundance of an individual star is not known a priori, so it is also a free parameter. With the stellar temperature set, we then derive the stellar radius from the absolute visual magnitude, for which we adopt values presented by Walborn et al. (2002). Since LH 10-3061 has not been observed in the $\mathrm{UV}$, we adopt a nominal wind terminal velocity of $3200 \mathrm{~km}$ $\mathrm{s}^{-1}$ for it, following prior UV analysis of Sk $-68^{\circ} 137$ (Prinja \& Crowther 1998); sources of terminal velocities for the other stars are given in notes to Table 1. Lacking the usual $\mathrm{H} \alpha$ wind diagnostic for most of the stars, we estimate mass-loss rates from the strength of He II $\lambda 4686$, although we recognize that as a less reliable indicator.

With regard to the helium abundance, generally $\mathrm{He} / \mathrm{H}=$ 0.1 (by number) is adopted unless noted otherwise. Potential nebular contamination of Balmer cores generally prevents accurate determinations of $\mathrm{He} / \mathrm{H}$ from intermediate-dispersion observations of $\mathrm{O}$ stars. For NGC 346-3, inspection of a high-resolution Anglo-Australian Telescope/UCLES observation (Walborn et al. 2000) supports $\mathrm{He} / \mathrm{H} \sim 0.1$. In contrast, the CTIO 4 m observation of LH 64-16 (Massey et al. 2000) analyzed here suggests a rather helium-rich chemistry, with $\mathrm{He} / \mathrm{H} \sim 0.25$. This same observation of LH 64-16 has been modeled independently by Massey et al. (2004), who find an even larger $\mathrm{He} / \mathrm{H}$ abundance ratio.

We estimate carbon and oxygen abundances from $\mathrm{C}$ IV $\lambda 4658$ and $\mathrm{O}$ IV $\lambda \lambda 3348-3349,3381-3410$, respectively, while all remaining elements are set to $0.4 Z_{\odot}$ (LMC) or $0.2 Z_{\odot}$ (SMC), following, e.g., Russell \& Dopita (1992). Solar abundances are taken from Grevesse \& Sauval (1998; $\log (\mathrm{C} / \mathrm{H})+12=8.52, \log (\mathrm{N} / \mathrm{H})+12=7.92)$, except that the oxygen abundance has recently been revised downward to $\log (\mathrm{O} / \mathrm{H})+12=8.66$ (Asplund 2003; Asplund et al. 2004).

\subsection{Results}

Early O giants in the Magellanic Clouds have previously been studied quantitatively by Puls et al. (1996), who derived stellar temperatures of 55, 60, and $60 \mathrm{kK}$ for NGC 346-3, Sk $-68^{\circ} 137$, and HDE 269810, respectively, from plane-parallel 

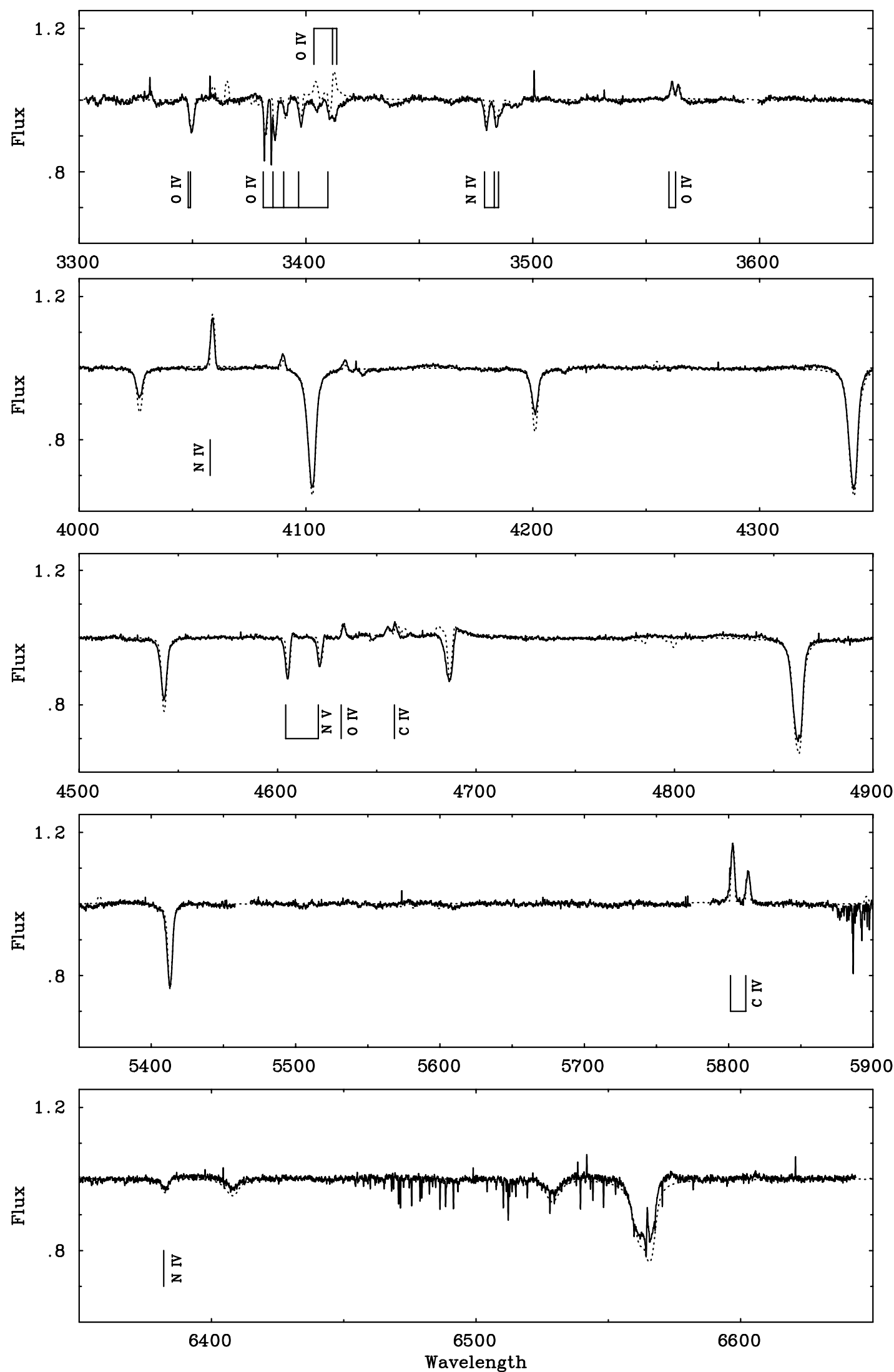

FIG. 5.-Atmospheric model fit (dotted lines) to the VLT/UVES high-resolution observation of HDE 269810 (solid lines). The spectral lines identified in each panel from top to bottom are $\mathrm{O}$ Iv $\lambda \lambda 3348,3349 ; 3381,3385,3390,3397,3410 ; 3404,3412,3414 ; 3560,3563$; and $\mathrm{N}$ iv $\lambda \lambda 3479,3483,3485 ; \mathrm{N}$ iv $\lambda 4058$; $\mathrm{N}$ v $\lambda \lambda 4604,4620, \mathrm{O}$ Iv $\lambda 4632$, and $\mathrm{C}$ iv $\lambda 4658 ; \mathrm{C}$ iv $\lambda \lambda 5801,5812 ;$ and $\mathrm{N}$ iv $\lambda 6381$. 
photospheric techniques, which neglected the effects of line blanketing. As with other recent redeterminations of $\mathrm{O}$ star temperature scales (e.g., Martins et al. 2002; Crowther et al. 2002), we find $2.5-5 \mathrm{kK}$ lower temperatures. Indeed, our results closely mimic those of Bouret et al. (2003) for NGC $346-3$, with stellar temperatures in the range $52.5 \mathrm{kK} \leq T_{\text {eff }} \leq$ $55 \mathrm{kK}$ and luminosities in the range $8 \times 10^{5} \leq \log \left(L / L_{\odot}\right) \leq$ $2.2 \times 10^{6}$, plus unclumped mass-loss rates of $1-2 \times 10^{-6} M_{\odot}$ $\mathrm{yr}^{-1}$ (Table 1).

More important in the present context are elemental abundances (see Table 1). For the LMC O2 giants, we find only modest increases in the nitrogen abundances from the ISM ratios. In contrast, large $\mathrm{N} / \mathrm{C}$ and $\mathrm{N} / \mathrm{O}$ ratios are obtained for the LMC ON2 giants, suggesting partially processed material at the stellar surfaces. For NGC 346-3 in the SMC, we confirm processed $\mathrm{CNO}$ abundances as derived by Bouret et al. (2003). Haser et al. (1998) previously estimated $\mathrm{N} / \mathrm{N}_{\odot} \geq 0.4$ and $\mathrm{C} / \mathrm{C}_{\odot} \leq 0.02$ for this star from UV spectral fits. Relative to their corresponding ISM ratios, we find that $\mathrm{N} / \mathrm{C}$ is enhanced in the atmospheres of LH 64-16, LH 10-3061, and NGC $346-3$ by factors of 46,46 , and 52 , and N/O by factors of 14,23 , and 12, respectively. These factors can be compared with those of the order of 100 derived by Crowther et al. (2002) and Hillier et al. (2003) for later type $\mathrm{O}$ supergiants in the Magellanic Clouds.

\subsection{Uncertainties}

In order to estimate the uncertainties of our abundance results, we have undertaken additional modeling of two kinds. First, the fits to the data discussed above have been reexamined to determine the range of parameters, if any, that might yield comparable or perhaps even improved fits. For example, in the models upon which the results of Table 1 are based, the $\mathrm{N}$ IV $\lambda 4058$ emission line was well matched, while the $\mathrm{N} v$ absorption lines were somewhat underpredicted. It was found that increasing the $T_{\text {eff }}$ of the two ON2 stars, LH 64-16 and LH 10-3061, to $56 \mathrm{kK}$ improves the fits to the $\mathrm{N} \mathrm{v}$ lines but somewhat overpredicts the $\mathrm{N}$ IV emission, as shown in Figure 4. Simultaneously, the fits to the very weak C IV $\lambda 4658$ emission line, the sole diagnostic of the carbon abundances in these data, were re-examined, with the result that $\mathrm{C} / \mathrm{C}_{\odot}$ in the $\mathrm{ON} 2$ stars could be reduced to as low as 0.02 . The only other possible changes indicated by this further modeling are small increases in $\mathrm{O} / \mathrm{O}_{\odot}$ for the two LMC O2 stars, HDE 269810 and Sk $-68^{\circ} 137$, to 0.6 and 0.7 , respectively. In particular, no changes to the derived $\mathrm{N}$ abundances are indicated. To summarize, as best we can determine from the intermediate-dispersion data alone, the uncertainties in $T_{\text {eff }}$ are $\pm 1 \mathrm{kK}$, in the carbon abundances a factor of 2.5 , and in the oxygen abundances a factor of 1.5. One could infer that the uncertainty in the nitrogen abundances is less than a factor of 1.5.

A second, more powerful comparison is provided by the available VLT/UVES high-resolution spectrogram of HDE 269810, with wavelength coverage extending into the yellowred. Not only are the multiple, weak $\mathrm{O}$ IV lines well resolved and defined in these data, additionally including $\lambda \lambda 3560,3563$ and 4632, but the strong $C$ IV $\lambda \lambda 5801,5812$ emission lines are available to better constrain the carbon abundance, and $\mathrm{N}$ IV $\lambda 6381$ is also available. The good fit obtained with the same models used to analyze the intermediate-dispersion data is shown in Figure 5. The only significant discrepancies are in the $\mathrm{O}$ IV $\lambda \lambda 3404,3412,3414$ triplet, which is predicted in emission by models this hot but is observed in absorption, and in higher members of the He II Pickering series, which remain overpredicted. The improved fit to hydrogen and other helium lines is noteworthy; the discrepancies at the lower dispersions are believed to be caused primarily by line broadening and resolution matching between the models and data. It is reassuring that the mass-loss rate based also on the $\mathrm{H} \alpha$ profile here agrees well with that determined previously from He II $\lambda 4686$ alone. The stellar parameters that differ in the high-resolution analysis are $T_{\text {eff }}, 55 \mathrm{kK} ; \log L / L_{\odot}, 6.38$; $\log \dot{M}\left(M_{\odot} \mathrm{yr}^{-1}\right),-5.5$; and $\mathrm{He} / \mathrm{H}, 0.07$; comparison with the respective values in Table 1 indicates the reliability of that analysis. The derived $\mathrm{C}, \mathrm{N}$, and $\mathrm{O}$ abundances relative to solar are $0.05,0.6$, and 0.8 , respectively; i.e., the $\mathrm{C}$ abundance is smaller by a factor of 4 , while the $\mathrm{N}$ and $\mathrm{O}$ abundances are larger by respective factors of 1.5 and 2 . It follows from these results that HDE 269810 already has an appreciable N/C enhancement over the LMC ISM by a factor of 23 .

The uncertainties in the solar CNO abundances given by Grevesse \& Sauval (1998) are $\pm 0.06 \mathrm{dex}$, or a factor of 1.15. The corresponding Magellanic Cloud ISM abundance uncertainties from Russell \& Dopita (1992) are 0.13-0.18 dex for C, $0.15-0.20$ dex for $\mathrm{N}$, and $0.06-0.10 \mathrm{dex}$ for $\mathrm{O}$, or average factors of $1.4,1.5$, and 1.2 , respectively. Thus, the uncertainties in the ratios are dominated by the stellar abundances, which we have estimated above to be factors of 2.5-4 for $\mathrm{C}$ and 1.5-2 for $\mathrm{N}$ and $\mathrm{O}$. The worst case is the $\mathrm{N} / \mathrm{C}$ ratio, which in principle could be uncertain by a factor of 6 . However, such a factor cannot apply randomly to the results, since it could invert the differential spectral morphology, which is not reasonable; on the other hand, a systematic effect of that magnitude cannot be ruled out with the present data. It is interesting that all of the $\mathrm{C}$ redeterminations are lower, while the $\mathrm{N}$ and $\mathrm{O}$ are somewhat higher. Thus, the $\mathrm{N} / \mathrm{C}$ ratios may be even larger than given in Table 1. Clearly it is highly desirable to reanalyze all of these stars with high-resolution data, at the same time determining their mass-loss rates from $\mathrm{H} \alpha$ and UV data, to refine the quantitative results.

\section{DISCUSSION: EVOLUTIONARY INTERPRETATIONS}

Curiously, the luminosities, and hence the masses, estimated by Walborn et al. (2002) for the O2 III(f*) stars HDE $269810\left(150 M_{\odot}\right.$, average of two methods) and Sk $-68^{\circ} 137$ $\left(100 M_{\odot}\right)$ are considerably higher than those for the ON2 III(f*) stars LH 64-16 (62 $\left.M_{\odot}\right)$, LH 10-3061 (81 $\left.M_{\odot}\right)$, and NGC 346-3 $\left(82 M_{\odot}\right)$. The opposite relationship might have been expected, since both mass-loss rates and mixing increase with mass (Meynet \& Maeder 2000). On the other hand, perhaps the longer evolutionary timescales at the lower masses favor the observability of mixing effects.

Until recently, these abundance results would have been interpreted simply in terms of more advanced evolutionary ages for the ON2 than the $\mathrm{O} 2$ objects, which remains a possibility. (Of course, mass transfer in a binary system is an alternative mechanism likely to be responsible for at least some members of the ON class; see Bolton \& Rogers 1978; Levato et al. 1988; Walborn \& Howarth 2000; Lennon 2003.) In the context of evolutionary models with rotation for massive stars (Maeder \& Meynet 2000; Meynet \& Maeder 2000), however, the degree of mixing of processed material to the surface at a given evolutionary age is a function of the initial rotational velocity. Indeed, $\mathrm{CNO}$ abundances in massive stars now become critical diagnostics of their initial parameters and evolutionary histories. For a given metallicity, the observed $T_{\text {eff }}$, $L$, and CNO abundances in principle enable derivation of the corresponding initial mass, current age, and initial rotational 


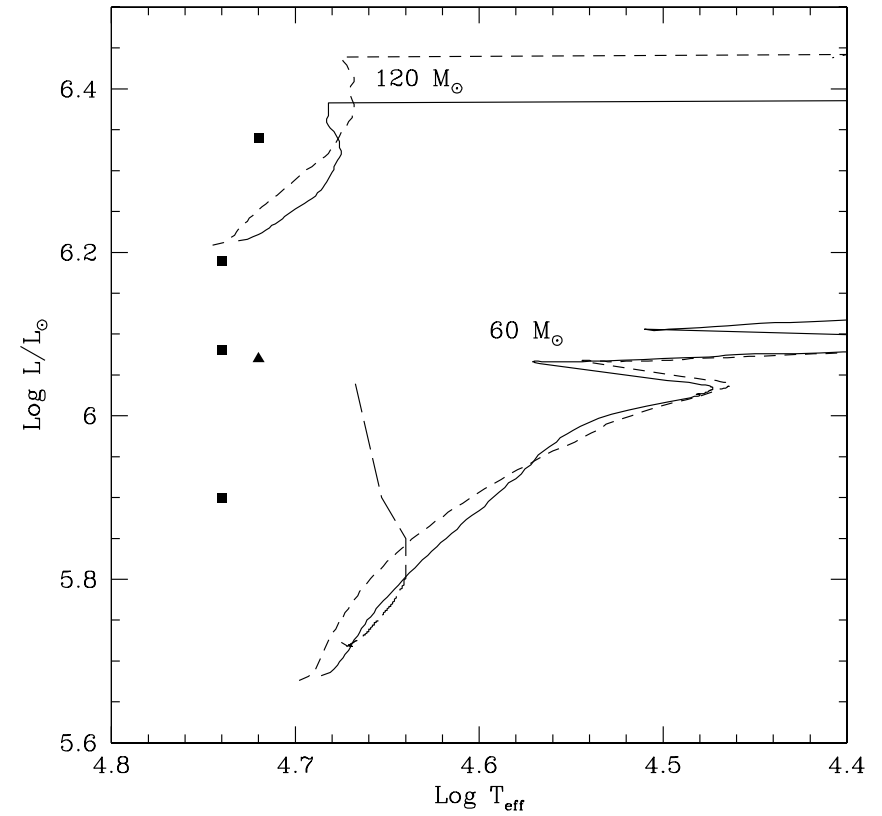

FIG. 6.-Theoretical H-R diagram comparing the observed stars with Geneva evolutionary tracks at both LMC and SMC metallicities for an initial rotational velocity of $300 \mathrm{~km} \mathrm{~s}^{-1}$. Also included is a $60 M_{\odot}$ homogeneous track at solar metallicity for an initial rotational velocity of $400 \mathrm{~km} \mathrm{~s}^{-1}$. Shown are LMC stars (squares), the SMC star (triangle), LMC tracks (solid lines), SMC tracks (short-dashed lines), and the homogeneous track (long-dashed line).

velocity. Complete grids of evolutionary tracks for the most massive stars, at Magellanic Cloud metallicities and with a range of rotational velocities, are currently under development in Geneva. Meanwhile, we display illustrative comparisons in Figures 6-8, courtesy of G. Meynet and A. Maeder.

Figure 6 is a theoretical HRD in which the locations of the stars discussed here are compared with Geneva evolutionary tracks for initial masses of 60 and $120 M_{\odot}$, at both LMC and SMC metallicities (mass fractions $Z=0.008$ and 0.004 , respectively) and with an initial equatorial rotational velocity of $300 \mathrm{~km} \mathrm{~s}^{-1}$. All of the stars lie near the zero-age main sequence (ZAMS), which is consistent with an early evolutionary stage for the two objects with normal spectra; the present spectroscopic analysis again indicates higher masses for them than for the ON2 objects. However, such a location is surprising for the latter, highly mixed stars. Their initial masses still place them above the Humphreys-Davidson limit, so they cannot be interpreted as post-red supergiants evolving blueward. A more relevant possibility may be that of "homogeneous" evolution along tracks that rise and curve blueward from the ZAMS in the HRD, which is predicted in the cases of strong mixing and/or very high initial rotational velocities (Langer \& El Eid 1986; Maeder 1987; Langer 1992; Meynet \& Maeder 2000). The $60 M_{\odot}$ homogeneous track at solar metallicity $(Z=0.020)$ with an initial rotational velocity of $400 \mathrm{~km} \mathrm{~s}^{-1}$ from the last reference is also reproduced in Figure 6; it trends near the locations of the ON2 III(f*) points. Indeed, Bouret et al. (2003) have concluded that such a track is required to understand NGC 346-3 as coeval with the other early $\mathrm{O}$ stars in the cluster.

Figures 7 and 8 compare our derived N/C and N/O ratios, respectively, relative to their corresponding interstellar media, taken at face value from Table 1, with the predictions of the same Geneva models. The N/C ratios in the ON2 III(f*) stars are the largest reported to date for near-main-sequence objects

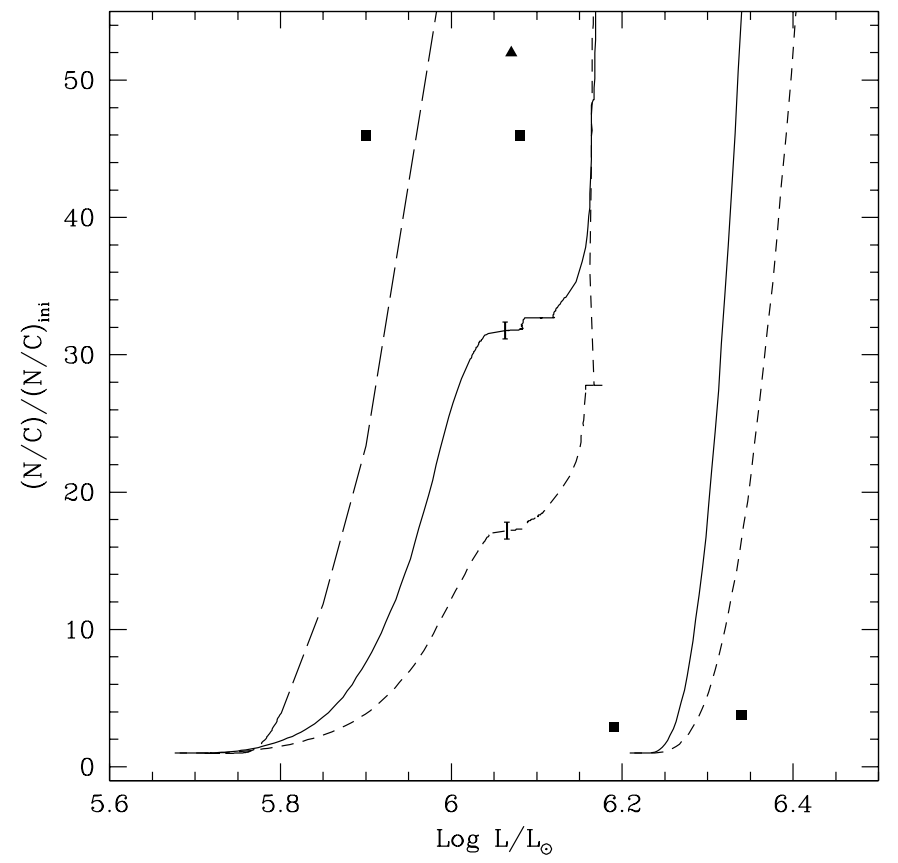

FIG. 7.-Derived stellar N/C abundance ratios relative to their initial (interstellar) values compared with predictions from the models in Fig. 6. The $60 M_{\odot}$ tracks are toward the left and the $120 M_{\odot}$ toward the right; short vertical line segments on the former indicate the end of the hydrogen-burning main sequence. Other symbols and line styles as in Fig. 6.

and are substantially larger than the standard model predictions, suggesting even more rapid mixing and perhaps providing additional support for homogeneous evolution. In contrast, the N/O ratios are compatible with the standard model predictions and indicate early operation of the $\mathrm{ON}$ cycle, as expected in only the most massive stars.

On the other hand, the even larger $\mathrm{N}$ enhancements derived for later type $\mathrm{O}$ supergiants in the Magellanic Clouds by Crowther et al. (2002) and Hillier et al. (2003) may imply that

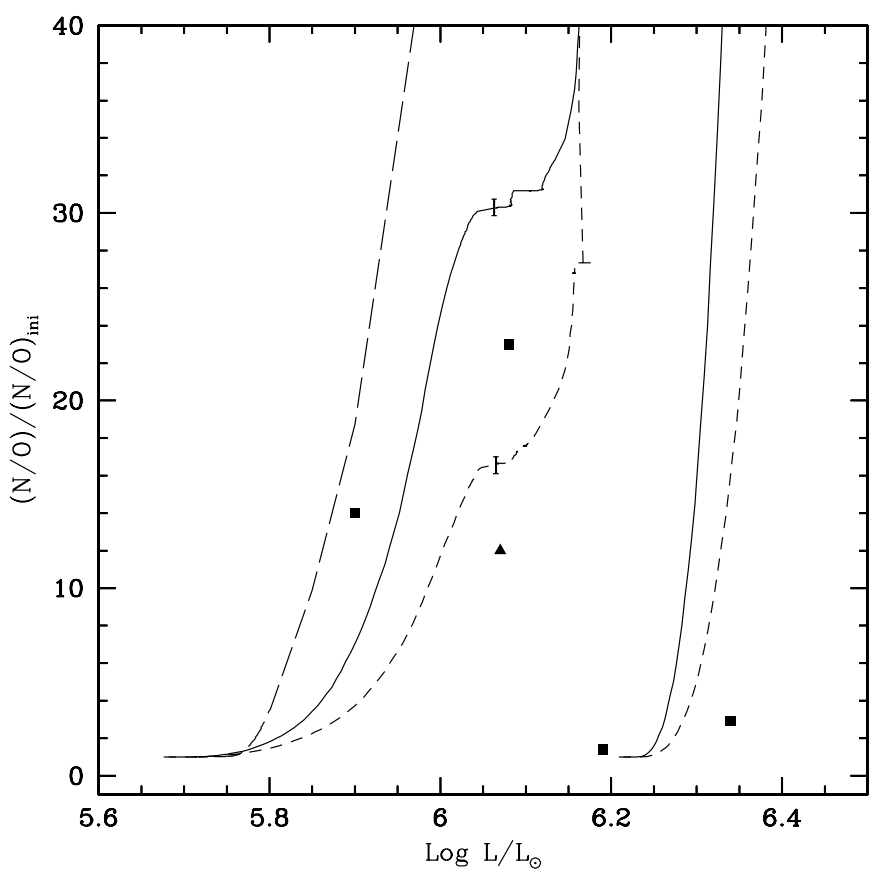

Fig. 8. - Same as Fig. 7, but for N/O abundance ratios. 
the ON2 III(f*) stars are simply predecessors of those objects along redward evolutionary tracks. Again, much stronger mixing effects near the main sequence than predicted by current models, at least for $\mathrm{N} / \mathrm{C}$, are then required by our observations. Further analysis of these phenomena, with improved data and relative to the latest models for rotating massive stars, will clarify these issues and provide an improved understanding of their post-main-sequence evolution.
We are very grateful to Georges Meynet and André Maeder for their substantial contributions to the discussion of these results, including Figures 6-8. We also thank the STScI Director's Discretionary Research Fund for publication support. P. M.'s participation was supported by NASA through grant GO-8633 from the Space Telescope Science Institute, which is operated by AURA, Inc., under contract NAS5-26555. We thank an anonymous referee for useful recommendations.
Asplund, M. 2003, in ASP Conf. Ser. 304, CNO in the Universe, ed. C. Charbonnel, D. Schaerer, \& G. Meynet (San Francisco: ASP), 275

Asplund, M., Grevesse, N., Sauval, A. J., Allende Prieto, C., \& Kiselman, D. 2004, A\&A, 417, 751

Bolton, C. T., \& Rogers, G. L. 1978, ApJ, 222, 234

Bouret, J.-C., Lanz, T., Hillier, D. J., Heap, S. R., Hubeny, I., Lennon, D. J., Smith, L. J., \& Evans, C. J. 2003, ApJ, 595, 1182

Conti, P. S. 1973, ApJ, 179, 181

Crowther, P. A., Hillier, D. J., Evans, C. J., Fullerton, A. W., De Marco, O., \& Willis, A. J. 2002, ApJ, 579, 774

Drissen, L., Moffat, A. F. J., Walborn, N. R., \& Shara, M. M. 1995, AJ, 110,2235

Grevesse, N., \& Sauval, A. J. 1998, Space Sci. Rev., 85, 161

Haser, S. M., Pauldrach, A. W. A., Lennon, D. J., Kudritzki, R.-P., Lennon, M., Puls, J., \& Voels, S. A. 1998, A\&A, 330, 285

Hillier, D. J., Lanz, T., Heap, S. R., Hubeny, I., Smith, L. J., Evans, C. J., Lennon, D. J., \& Bouret, J. C. 2003, ApJ, 588, 1039

Hillier, D. J., \& Miller, D. L. 1998, ApJ, 496, 407

Langer, N. 1992, A\&A, 265, L17

Langer, N., \& El Eid, M. F. 1986, A\&A, 167, 265

Lanz, T., \& Hubeny, I. 2003, ApJS, 146, 417

Lennon, D. J. 2003, in IAU Symp. 212, A Massive Star Odyssey, ed. K. van der Hucht, A. Herrero, \& C. Esteban (San Francisco: ASP), 308

Levato, H., Morrell, N., Garcia, B., \& Malaroda, S. 1988, ApJS, 68, 319

Maeder, A. 1987, A\&A, 178, 159

\section{EFERENCES}

Maeder, A., \& Conti, P. S. 1994, ARA\&A, 32, 227

Maeder, A., \& Meynet, G. 2000, ARA\&A, 38, 143

Martins, F., Schaerer, D., \& Hillier, D. J. 2002, A\&A, 382, 999

Massey, P., Bresolin, F., Kudritzki, R.-P., Puls, J., \& Pauldrach, A. W. A. 2004, ApJ, 608, 1001

Massey, P., Waterhouse, E., \& DeGioia-Eastwood, K. 2000, AJ, 119, 2214

Meynet, G., \& Maeder, A. 2000, A\&A, 361, 101

Prinja, R. K., \& Crowther, P. A. 1998, MNRAS, 300, 828

Puls, J., et al. 1996, A\&A, 305, 171

Russell, S. C., \& Dopita, M. A. 1992, ApJ, 384, 508

Smith, K. C., \& Howarth, I. D. 1994, A\&A, 290, 868

Walborn, N. R. 1976, ApJ, 205, 419

1988, in IAU Colloq. 108, Atmospheric Diagnostics of Stellar

Evolution, ed. K. Nomoto (Berlin: Springer), 70

2001, in ASP Conf. Ser. 242, Eta Carinae and Other Mysterious Stars,

ed. T. Gull, S. Johansson, \& K. Davidson (San Francisco: ASP), 217

2003, in ASP Conf. Ser. 304, CNO in the Universe, ed. C. Charbonnel,

D. Schaerer, \& G. Meynet (San Francisco: ASP), 29

Walborn, N. R., \& Howarth, I. D. 2000, PASP, 112, 1446

Walborn, N. R., Lennon, D. J., Haser, S. M., Kudritzki, R.-P., \& Voels, S. A. 1995, PASP, 107, 104

Walborn, N. R., Lennon, D. J., Heap, S. R., Lindler, D. J., Smith, L. J., Evans, C. J., \& Parker, J. W. 2000, PASP, 112, 1243

Walborn, N. R., et al. 2002, AJ, 123, 2754 